\title{
Le projet SOLEIL
}

\author{
Arteferétilished by EDP Sciences and available at http://www.annphys.org \\ or http://dx.doi.org/10.1051/anphys/1997002 \\ CEADSMIDRECAM, Service des Photons, Atomes et Molécules, Bâtiment 522, Centre \\ d'Études de Saclay, 91191 Gif-sur-Yvette cedex, France
}

\begin{abstract}
Résumé : Le projet SOLEIL est un ensemble de sources de lumière (anneau de stockage pour le rayonnement synchrotron, laser à électrons libres ultra-violet sur anneau, laser à électrons libres infrarouge sur accélérateur linéaire) qui doit remplacer les installations du laboratoire LURE (Orsay) à partir de 2003. Les caractéristiques uniques des faisceaux de photons accordables de l'infra-rouge jusqu'aux rayons $X$ durs (haut flux, grande brillance, facilite d'accès à une polarisation linéaire et circulaire, faible divergence, structure temporelle spécifique), expliquent que les applications potentielles se situent dans une vaste palette scientifique de la recherche fondamentale et finalisée développée tant à l'Université que dans les organismes publics et dans l'industrie.
\end{abstract}

\section{HISTORIQUE}

Le rayonnement synchrotron est constitué par les faisceaux de photons produits par les particules (électrons ou positrons) accélérés dans des anneaux de stockage ; le domaine spectral de la lumière émise dépend de l'énergie des particules et couvre continûment tout le spectre électromagnétique de l'infra-rouge aux rayons $\mathrm{X}$ durs avec des caractéristiques uniques en haut flux, grande brillance, facilité d'accès à une polarisation linéaire et circulaire, faible divergence et structure temporelle spécifique. L'interêt et la complémentarité de ces sources par rapport aux sources conventionnelles de laboratoire (tubes à rayons $\mathrm{X}$, lampes à décharges et sources lasers) est à l'origine de la demande et de l'utilisation de ce rayonnement dans l'ensemble des pays développés pour une grande variété d'applications aussi bien en recherche fondamentale que finalisée.

En France, au début des années 1970, sous l'impulsion de scientifiques pionniers, a démarré et s'est développé régulièrement l'utilisation des faisceaux de l'anneau de stockage ACO (Anneau de Collision d'Orsay) puis plus tard d'un autre anneau DCI (Dispositif de Collisions dans l'Igloo), machines conçues pour la Physique des Hautes Energies.

Le Laboratoire LURE (Laboratoire pour l'Utilisation du Rayonnement Electromagnétique) devait naître quelques années après pour fournir à la communauté nationale française des faisceaux de lumière synchrotron pour une large gamme de disciplines scientifiques. Dans les années 1980, la forte demande des utilisateurs et la concurrence internationale a justifié que la France se dote d'une machine dédiée et

(1) Vice-Présidente du Conseil Scientifique SOLEIL 
optimisée pour le rayonnement synchrotron : SuperACO à Orsay (en remplacement $\mathrm{d}^{\prime} \mathrm{ACO}$ ) pour le domaine des rayons $\mathrm{X}$ mous, l'ultra-violet lointain et l'infra-rouge et participe à la construction et l'utilisation d'une machine Européenne : l'ESRF (European Synchrotron Radiation Facility) à Grenoble pour le domaine des rayons X durs. Aujourd'hui la demande annuelle à de LURE additionnée de la part française à l'ESRF concerne quelques 1800 à 2000 utilisateurs de près de 500 laboratoires.

Depuis le début des années 1990, la communauté scientifique française a pris progressivement conscience de son retard par rapport aux autres pays en émettant l'idée d'une nouvelle machine pour la France [1]. Parallèlement, la demande pressante et continue à LURE et l'ESRF ainsi que les besoins manifestés à l'occasion de différents colloques à Orléans (Mars 1994), Aix en Provence (Avril 1994) et Orsay (Avril 1996), le mûrissement du projet SOLEIL [2-4], enfin l'obsolescence des machines de LURE révélée par l'Audit de ce laboratoire demandé par le Ministère de la Recherche ont attiré l'attention des responsables des grands organismes et leur ministère de tutelle (Conseil des très grands Equipements depuis 1992) sur la nécessité de mettre en chantier une machine de nouvelle génération apte à remplacer les installations de LURE devenues obsolètes afin que les besoins non couverts par l'ESRF puissent être satisfaits.

Depuis, une convention entre le CNRS et le CEA signée le 2 mai 1996, a mis en place une nouvelle structure chargée de l'Avant Projet Détaillé du projet SOLEIL et ce pour une durée de trois ans; elle est composée d'un Conseil, d'un Conseil Scientifique et d'une équipe projet dirigée par un Chef de Projet chargé, entre autres, de définir le cahier des charges du nouveau laboratoire, d'estimer les coûts de construction et d'exploitation, de proposer un calendrier, de proposer un statut juridique pour le laboratoire et de préciser les exigences relatives au site.

\section{DESCRIPTIF TECHNIQUE}

La source est un anneau de stockage à électrons (avec options positrons) de $2,15 \mathrm{GeV}$ injecté par un ensemble accélérateur linéaire et d'un élanceur (ou booster) de $200 \mathrm{McV}$ (voir figure 1). L'anneau produit un rayonnement dans la gamme d'énergie de $10 \mathrm{eV}$ à 20 $\mathrm{keV}$ grâce à des insertions magnétique périodiques dans les sections droites : ondulcurs $(10 \mathrm{eV}$ à $2 \mathrm{keV})$ et wigglers $(10 \mathrm{eV}$ à $20 \mathrm{keV})$ ainsi que dans les aimants dipolaires de courbure $(10 \mathrm{eV}$ à $20 \mathrm{keV})$. L'anneau a une circonférence de $336 \mathrm{~m}$ ct peut accucillir plus de quarante lignes de lumière dont 14 issues des onduleurs ou wigglers. L'anneau comporte 4 sections droites de grande longucur $(14 \mathrm{~m})$ dont l'une sera équipée d'un élément d'insertion magnétique complexe" pour un laser à électrons libres qui fournira une lumière cohérente spatialement et temporellement entre 350 et $150 \mathrm{~nm}$ (voire $100 \mathrm{~nm}$ ), picoseconde, en synchronisation naturelie avec le rayonnement synchrotron. Trois types de fonctionnement ont été définis avec une durée de vie moyenne des faisceaux toujours supérieure à 10 heures et pouvant alteindre 20 heures ou plus:

i) mode haute brillance pour lequel le taux de répétition est si élevé que les faisceaux sont en fait une source quasi-continue ; des brillances ultimes supérieures à $10^{20}$ photons $/ \mathrm{s} / \mathrm{mm}^{2} / \mathrm{mrad}^{2} / 0,1 \%$ bande passante sont attendues dans certains onduleurs et plaçant ainsi la machine SOLEIL au plus haut niveau de la compétition internationale. 
ii) mode structure temporelle avec un taux de répétition beaucoup plus faible et des impulsions utilisables de 50 ps séparées de $140 \mathrm{~ns}$ pour des études cinétiques pico et nanaoseconde et permettant de synchroniser avec des lasers extérieurs.

iii) mode laser à électrons libres à $1,5 \mathrm{GeV}$ compatible avec le mode structure temporelle et donc permettant entre autres des expériences à deux couleurs LEL rayonnement synchrotron.

Des faisceaux à polarisation ajustable : linéaire et direction du champ électrique variable, circulaire gauche ct droite, seront disponibles grâce à des insertions magnétiques particulières.

Enfin, le projet prévoit, pour le moment comme option, l'installation à proximité immédiate de la machine, d'un ensemble de deux lasers à électrons libres dans l'infrarouge proche et lointain couvrant la gamme de longueur d'onde de $2 \mu \mathrm{m}$ à $2 \mathrm{~mm}$ et synchronisables avec les photons de SOLEIL.

\section{COMPARAISON AVEC QUELQUES SOURCES LASER}

L'augmentation récente des performances des sources laser dans le domaine des rayons $\mathrm{X}$ mous justifient une comparaison avec celles des sources de rayonnement synchrotron et en particulier celles de SOLEIL. La figure 1 montre le flux comparé en nombre de photons par seconde et par $0,1 \%$ de bande passante et la puissance moyenne en watt. Les sources laser examinées ont été limitées au laser à électrons libres [5], les sources cohérentes par génération d'harmoniques [6]. les sources laser-plasma [7], et les lasers X [8]. La figure 1 montre la supériorité des sources synchrotron sur les sources laser principalement à cause de leur taux de répétition élevé et leur accordabilité. Dans I'UV proche et lointain, le laser à électrons libres est particulièrement attractif. La figure 2 montre l'éclairement comparé de ces sources, défini comme la puissance crête par unité de surface de la tache focale obtenue éventuellement derrière une optique. Dans ce cas et contrairement au flux, les sources laser ( $y$ compris le laser à électrons libres) sont très performantes par rapport aux sources synchrotron, principalement à cause de leur largeur d'impulsion dans le domaine femtoseconde qui rend leur puissance crête très élevée et donc permettent l'accès à la détection de phénomènes non linéaires.

L'utilisation dominante du RS comme source quasi-continue totalement accordable en longueur d'onde et celle des sources laser comme sources à très courtes impulsions et très haute puissance crête démontre de manière évidente la complémentarité de leur usage. D'autres différences importantes (non détaillées ici, voir ref. [9]) entre ces sources concernent la cohérence spatiale et temporelle, les propriétés spectrales, la largeur d'impulsion et le taux de répétition, la polarisation et doivent guider les utilisateurs potentiels de ces sources pour leurs besoins spécifiques.

Les progrès des sources laser se manifestent par un développement dans le domaine ultra-violet sous vide et rayons $\mathrm{X}$ mous. C'est particulièrement frappant pour le cas des laser-plasma et les lasers $\mathrm{X}$. Pour le rayonnement synchrotron, les progrès réalisés sur les machines de troisième génération et celles des optiques de focalisation ont accru les propriétés de cohérence spatiale, voire de cohérence temporelle, les rapprochant ainsi des sources laser. Enfin, les lasers à électrons libres ultra-violet se développent avec des performances aussi bonnes en puissance moyenne qu'en puissance crête dans l'ultraviolet, comblant ainsi le « gap » entre les sources laser classiques et le RS. 


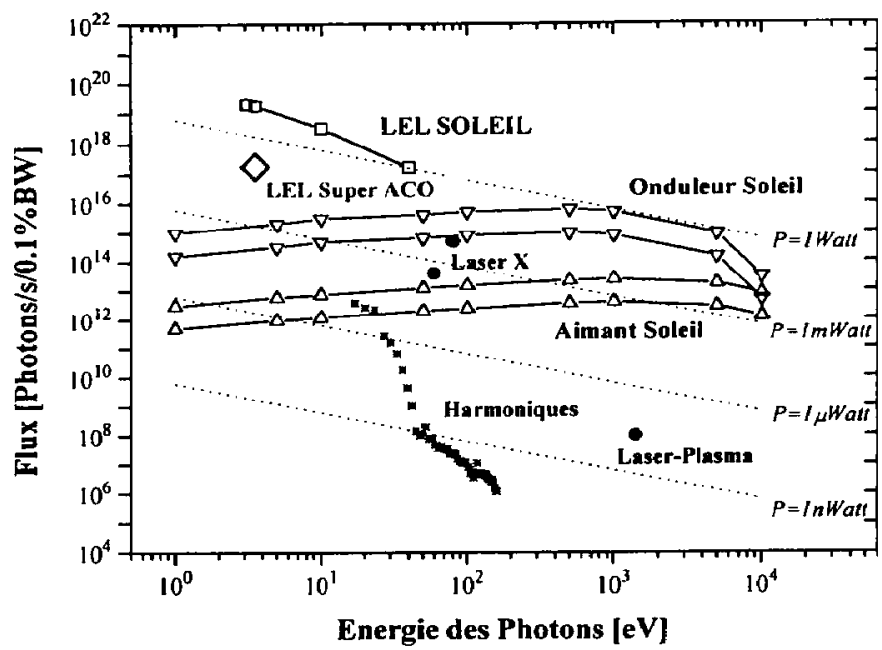

Figure 1 : Flux moyen comparé des sources Aimants et onduleurs SOLEIL (cas des deux modes de fonctionnement "haute brillance" et "structure temporelle") avec le laser à électron libres UV sur SuperACO et sur SOLEIL, des sources laser par génération d'harmoniques, des lasers $\mathrm{X}$ et des sources laseriplasmas.

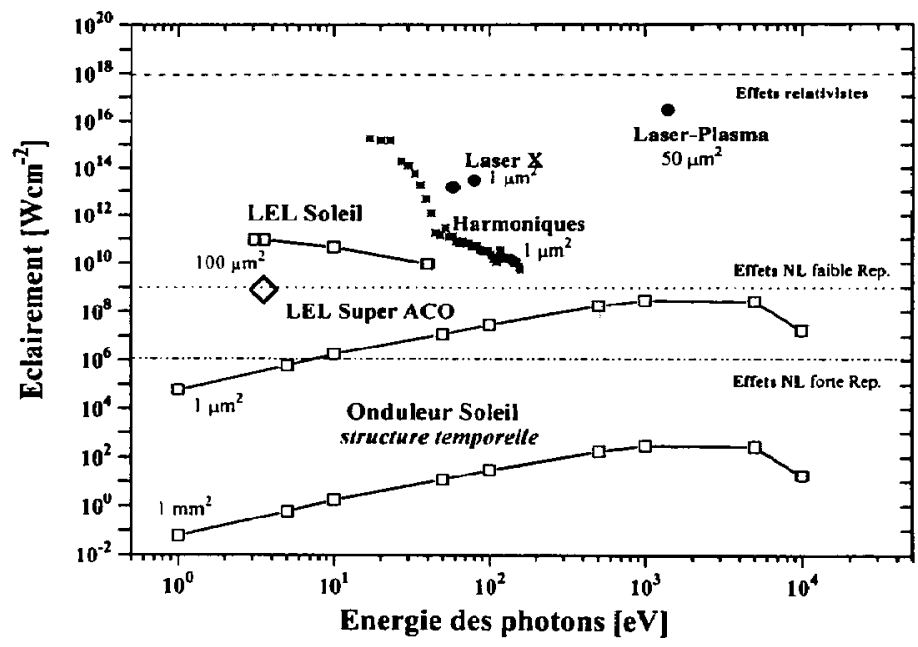

Figure 2 : Eclairement comparé des sources onduleurs SOLEIL (cas du mode de fonctionnement "structure temporelle") avec le laser à électron libres UV sur SuperACO et sur SOLEIL, des sources laser par génération d'harmoniques, des lasers $\mathrm{X}$ et des sources laser/plasmas. La surface de la tache focale obtenue avec une optique appropriée est indiquée pour chaque source. Les seuils de détection approximatifs des effets non linéaires (NL) pour les sources de faible et fort taux de répétition ainsi que les effets relativistes sont mentionnés. 


\section{APPLICATIONS POTENTIELLES}

Les spécificités du rayonnement synchrotron ont révolutionné depuis les années 70 , nombre de techniques expérimentales basées sur l'interaction rayonnement-matière dans de nombreuses disciplines scientifiques en recherche fondamentale comme la physique atomique et moléculaire, la physique de l'état condensé (surfaces et solides), la chimie du solide, la biologie et la géochimie pour ne citer que les plus importantes. L'interêt d'un tel outil en recherche finalisée s'est révélé rapidement à cause des besoins en analyse et de caractérisation structurale, électronique et magnétique de nombreux matériaux.

La technologie moderne qui évolue de plus en plus vers une miniaturisation des systèmes comme l'intégration des composants électroniques, obtention de matériaux magnétiques à haute densité d'enregistrement, films minces supra-conducteurs à haute température critique, catalyseurs très divisés etc..Cela nécessite de travailler à très petite échelle. De plus, le rôle des surfaces et interfaces au niveau atomique des systèmes miniaturisés, élaborés artificiellement à partir de composés différents, devient prépondérant. Par ailleurs, les matériaux intéressants pour leur fonction ou propriété macroscopique, sont complexes dans leur composition, texture, état physique, ou réactivité. La corrélation de leurs propriétés avec leur caractérisation chimique, structurale, électronique et magnétique au niveau atomique et mésoscopique est primordiale. C'est le cas dans de nombreuses situations comme le contrôle durant l'élaboration d'un matériau sous vide, la mise au point de certains procédés de fabrication, le contrôle qualité, les effets de vieillissement, les effets de contraintes extérieures (chimie, température, pression, champ éłectrique, radiations) et généralement les études visant à établir les relations structure-propriétés ou structure-fonctionalité.

D'autres besoins qui devraient connaître un fort développement dans le futur concernent la fabrication de micro-objets par lithographie par rayons X, la métrologie de détecteurs et d'optiques dans le domaine des rayons $\mathrm{X}$, des études spécifiques sur les plasmas laser et sur la physique atomique associée. Sont concernés à des titres divers, les grands programmes nationaux : défense (Physique autour du projet laser megajoule), Santé (médicaments, Energie (Retraitement et stockage des déchets nucléaires, matériaux pour les procédés d'enrichissement isotopique), Environnement (pollution atmosphérique et des sols) ou technologiques concernant de nombreux secteurs industriels (chimie fine, pétrochimie, électrochimie, électronique, plastiques, automobile, emballage, verres, pharmacie, cosmétique, agro-alimentaire).

\subsection{Accordabilité}

L'accordabilité en longueur d'onde dans des régions spectrales inaccessibles avec des sources de laboratoire (tubes à rayons $\mathrm{X}$, lasers, lampes à décharge) constitue la caractéristique de base du rayonnement synchrotron. Elle permet par le choix quasiillimité du niveau électronique de presque chaque élément du tableau périodique, d'obtenir la sélectivité chimique dans l'étude des propriétés structurales et électroniques de la matière, à l'échelle atomique. En effet, chaque élément $Z$ absorbe la lumière selon un spectre caractéristique de son numéro atomique. De nombreuses techniques sont associées au rayonnement synchrotron et ont été évaluées par rapport à de nombreuses méthodes concurrentes [10], mais seules les grandes lignes sont présentées ici. 
En effet, les techniques d'absorption, de diffraction et de diffusion des rayons $\mathrm{X}$ avec toutes ses variantes en méthodes de détection permettent d'obtenir l'ordre structural respectivement à courte et grande distance dans pratiquement n'importe quel échantillon, indépendamment de son état physique ou de sa complexité, grâce à l'accordabilité du rayonnement. Sont étudiés couramment des matériaux amorphes ou cristallins, en alliages, en couches minces, en nanophases, des surfaces et interfaces, des composés moléculaires et biologiques, liquides, des solutions, des colloïdes, des gaz etc...

La photoémission, technique privilégiée pour l'étude des propriétés électroniques de la matière, associée à une longueur d'onde des photons variable permet non seulement de bénéficier de la sélectivité chimique mais aussi en minimisant le libre parcours des électrons, obtenir une sensibilité maximale pour les atomes de surface par rapport à ceux du volume.

La fluorescence dans le domaine des $\mathrm{X}$ mous et de l'ultra-violet lointain, technique complémentaire de la photoémission, devient un outil privilégié avec le choix de la longueur d'onde de la lumière excitatrice pour sonder les propriétés électroniques de la matière en volume. Elle permet aussi dans le domaine $\mathrm{X}$ de fournir un outil exceptionnellement sensible pour l'analyse de traces.

La spectrométrie de masse associée à la spectroscopie photoélectronique et/ou la fluorescence par des méthodes de coïncidence appropriées permet d'aborder les phénomènes fondamentaux de l'ionisation et de la fragmentation moléculaire [11], à partir d'espèces isolées (molécules, agrégats) ou adsorbées sur des surfaces, dans des états électroniques spécifiques.

\subsection{Le flux et la brillance}

Le flux et la brillance du rayonnement synchrotron $\left(10^{12}\right.$ fo is plus forte dans le domaine $\mathrm{X}$ que les tubes à rayons $\mathrm{X}$ conventionnels pour une source comme SOLEIL) permettent de réaliser des expériences inconcevables antérieurement soit par la "rareté" de l'espèce étudiée, c'est à dire de faible densité comme des objets en phase gazeuse : ions, états excités, radicaux, agrégats libres, ou des échantillons très petits (cristaux biologiques, inclusions dans des métaux, éléments traces, dopants dans des matrices, couches minces etc...On peut aussi concevoir de très nombreuses expériences permettant d'associer des informations spectroscopiques (électroniques, magnétiques) et structurales en "imagerie" avec une résolution latérale sub-micronique.

La brillance permettra de rendre la méthode de fluorescence $X$ particulièrement performante pour l'analyse de traces puisque la limite de sensibilité obtenue avec des sources de 3 ième génération atteint l'attogramme.

La brillance permet d'obtenir plus aisement une haute résolution spectrale surtout dans le domaine de l'ultra-violet et des rayons $X$ mous et ouvre des perspectives complètement nouvelles. On peut par exemple envisager d'accéder à des états électroniques directement responsables des propriétés des solides à l'échelle des énergies thermiques (chaleur spécifique, transport, magnétisme, transitions de phase.....) ou de la supra-conductivité (à l'échelle du gap).

Le flux et la brillance rendent aussi possible de suivre en temps réel des mécanismes réactionnels et les transformations de phase de matériaux soumis à des contraintes extérieures (température, pression, champ électrique) ou au cours de réactions chimiques. Les méthodes structurales (absorption et diffraction) mentionnées ci-dessus sont des outils particulièrement puissants. 


\subsection{Polarisation}

La polarisation linéaire et circulaire offre une dimension supplémentaire pour la caractérisation des propriétés magnétiques des surfaces, des interfaces de matériaux nouveaux et de basse dimensionnalité. Les règles de sélection de l'interaction photonmatière font que, lorsqu'on a choisi la longueur d'onde d'excitation avec la double sélectivité chimique et orbitalaire, et la polarisation (linéaire parallèle et perpendiculaire, circulaire droite ou gauche) on peut sonder l'état de spin et l'orientation des moments magnétiques porté par l'atome sélectionné et responsable de l'état ferro, antiferro ou ferrimagnétique du matériau.

Les méthodes comme l'absorption des rayons $\mathrm{X}$, la diffraction $\mathrm{X}$ et les méthodes de photoémission ou de fluorescence $X$ utilisant la polarisation et la brillance $\mathrm{du}$ rayonnement fournissent ces informations avec la sensibilité surface/volume et aussi avec une résolution latérale au niveau micronique, ce qui correspond à la taille des "bits" magnétiques visée pour le futur.

Le choix de la polarisation du rayonnement permet aussi d'accéder aux propriétés électroniques et structurales de systèmes anisotropes (surfaces, interfaces, films minces, molécules chirales).

\subsection{La cohérence}

A priori, les sources de rayonnement synchrotron sont beaucoup moins cohérentes que les lasers, mais ces dernières années ont vu de grands progrès dans ce domaine, en rayonnement synchrotron. En effet, les propriétés de cohérence de la lumière d'un onduleur sur les nouvelles machines sont particulièrement intéressantes à cause de la faible l'émittance de la source (qui définit la cohérence transverse) et, derrière un monochromateur, à la pureté spectrale du faisceau (qui définit la cohérence temporelle). Les longueurs de cohérence sont de l'ordre du micron (tranverse) à quelques dizaines de microns (temporelle).

La diffusion de la lumière cohérente par un milieu désordonné fournit une figure de diffraction appelée "speckle" reflétant le désordre local. Si celui-ci évolue, on peut observer les fluctuations d'intensité et accéder directement à la dynamique du système. Les applications visées avec SOLEIL concerne le domaine des rayons $\mathrm{X}$ mous en matière molle (cristaux liquides, polymères, surfactants, collö̈des). Le flux cohérent sera tel que l'on peut espérer mesurer la dynamique de fluctuation dans le domaine de la microseconde dans des transitions de phase.

La cohérence permet aussi d'utiliser la technique de contraste de phase pour obtenir des images avec une résolution exceptionnelle et ceci pour des éléments lćgers et pour des objets de taille supérieure à $10 \stackrel{\circ}{\AA}$.

D'autres applications concernent la mesure d'indices, de réflectivité complexe, l'holographie tomographique etc...

\subsection{La structure temporelle}

Le mode de fonctionnement «structure temporelle" en réduisant le nombre de « paquets» de particules stockés dans la machine abaisse le taux de répétition de la 
lumière synchrotron à quelques $\mathrm{MHz}$ et rend les impulsions de quelques dizaines de picoseconde du rayonnement synchrotron utilisable.

L'analyse temporelle de la fluorescence en intensité et polarisation, dans des systèmes photoexcités dans l'ultra-violet proche est un outil de choix en biologie, car cette méthode permet d'accéder à des informations structurales d'objets non cristallisés, en solution ou dans un milieu complexe.

Le caractère pulsé peut aussi être mis à profit en spectroscopie photoélectronique par temps de vol ou par ionisation par champ ou encore en spectrométrie de masse en mode de détection en multicoïncidences. C'est en physique moléculaire que ces techniques sont exploités pour la spectroscopie à haute résolution d'ions moléculaires, d'agrégats et la dynamique d'états excités en couche de valence ou en couche interne.

Les expériences à deux couleurs combinant laser et rayonnement synchrotron requièrent l'utilisation du caractère pulsé du rayonnement. Le mode de pompe-sonde permet l'accès aux états excités non seulement en physique atomique et moléculaire mais aussi en physique des surfaces et en photobiologie. Outre les lasers pulsés conventionnels, le laser à électrons libres (optimisé dans l'UV) naturellement synchronisé avec le rayonnement synchrotron dans tous le domaine spectral, peut être couplé avec lui en utilisant la fluorescence, la spectrométrie de masse ou la photoémission comme moyen de détection. D'autres expériences combinant le laser à électrons libres dans l'infra-rouge et le rayonnement synchrotron en synchronisme temporel ou non seront aussi possibles pour diverses applications : électrochimie, photochimie, physico-chimie de surfaces ......

\section{CONCLUSIONS}

Le projet SOLEIL constitue donc un ensemble de sources de lumière avec des performances tout à fait exceptionnelles, dans tout le domaine électromagnétique qui offrira à la communauté scientifique des possibilités extrêmement variées dont certaines ne sont pas encore identifiées aujourd'hui, tant les frontières des domaines scientifiques et technologiques potentiellement intéressés sont encore floues. La capacité de la machine devra satisfaire les besoins des utilisateurs actuels de LURE et bien d'autres qui se sont manifestés au cours de la prospective scientifique de ces dernières années. Enfin un effort important sera consenti pour organiser le laboratoire avec les structures adéquates pour sa mission de service sur un rythme annuel de l'ordre de 5000 heures, tout en préservant un espace pour la recherche propre du laboratoire.

\section{Remerciements}

Je remercie J.L. Laclare (Chef du Projet), D. Chandesris et P. Morin (co-Responsables du programme expériences), Y. Petroff (Président du Conseil Scientifique) et les nombreux autres collègues participant au projet, pour avoir mis à disposition l'ensemble des documents du projet SOLEIL publiés et non publiés, qui a permis l'élaboration de cet article. Je remercie aussi F. Fabre (Directeur-Adjoint de LURE) pour la lecture critique du manuscrit. 


\section{Références}

[1] Avant-Projet SOLEIL J. Lecante (Editeur), Rapport Interne LURE (1991)

[2] le Projet SOLEIL : Argumentation Scientifique, ed. par D. Chandesris, P. Morin et I. Nenner, Les Editions de Physique (1993)

[3] Le projet SOLEIL : Etude technique complète, Rapport interne LURE (1994)

[4] Le projet SOLEIL, version abrégée, Rapport interne LURE (1995)

[5] M.E. Couprie, Colloque UVX94, Annales de Physique, C1 Suppl. 5, 19 (1994)

[6] A. L'Huillier et al. Int. J. Non Linear optical Physics and Mat. 4, 7467 (1995)

[7] J.C. Gauthier, P. Audebert, S. Bastiani, J.P. Geindre, A. Dos Santos, G. Grillon, A. Mysyrowicz, A. Antonetti, Colloque UVX96, ce volume et refs. Incl.

[8] Ph. Zeitoun, F. Albert, A. Carillon, P. Jaeglé, G. Jamelot, A. Klisnick, S. Sebban, D. ros et B. Rus, Colloque UVX96, ce volume et refs. Incl

[9] M.E. Couprie, F. Fabre, L. Nahon, I. Nenner, D. Normand, Annales de Physique (soumis 1996)

[10] l. Nenner, J. Doucet et H. Dexpert, Les techniques de l'Ingénieur, P 2700, 7 (1996)

[11] I. Nenner et P. Morin, Electronic and nuclear relaxation of core excited molecules

in "VUV and soft X-ray Photoionization studies in atoms and molecules" U. Becker and D.A. Shirley eds., Chapter 9, pp291-354, Plenum Publish. London (1996) 